\title{
A Mixed-Integer Second-Order Cone Programming Algorithm for the Optimal Power Distribution of AC-DC Parallel Transmission Channels
}

\author{
Shunjiang Lin *(D), Zhibin Yang, Guansheng Fan, Mingbo Liu, Sen He, Zhiqiang Tang and \\ Yunong Song \\ School of Electric Power Engineering, South China University of Technology, Guangzhou 510640, China \\ * Correspondence: linshj@scut.edu.cn; Tel.: +86-135-8049-8302
}

Received: 20 July 2019; Accepted: 16 September 2019; Published: 20 September 2019

\begin{abstract}
For the controllability of the transmission power of DC transmission channels, the optimal power distribution (OPD) of AC-DC parallel transmission channels is an effective measure for improving the economic operation of an AC-DC interconnected power grid. A dynamic optimal power flow model for day-ahead OPD of AC-DC parallel transmission channels is established in this paper. The power flow equation constraints of an AC-DC interconnected power grid and the constraints of the discrete regulation requirement of the transmission power of DC channels are considered, which make the OPD model of the AC-DC parallel transmission channels a mixed-integer nonlinear non-convex programming (MINNP) model. Through a cone relaxation transformation and a big $M$ method equivalent transformation, the non-convex terms in the objective function and constraints are executed with the convex relaxation, and the MINNP model is transformed into a mixed-integer second-order cone programming model that can be solved reliably and efficiently using the mature optimization solver GUROBI. Taking an actual large-scale AC-DC interconnected power grid as an example, the results show that the OPD scheme of the AC-DC parallel transmission channels obtained by the proposed algorithm can effectively improve the economical operation of an AC-DC interconnected power grid.
\end{abstract}

Keywords: AC-DC interconnected power grid; optimal power distribution of transmission channels; dynamic optimal power flow; cone relaxation; big $\mathrm{M}$ method; mixed-integer second-order cone programming

\section{Introduction}

In recent years, to deal with the issues of uneven distribution of power sources and loads in different regions, long-distance and large-capacity ultra/extra-high voltage transmission techniques have been widely applied to power grids in China. Large-scale AC-DC interconnected power grids have been formed in several regional power grids such as the China Southern Power Grid and the East China Power Grid [1-4]. In an AC-DC interconnected power grid, there are several AC and DC transmission channels to transmit electric energy among different regions. Due to the difference of line resistance and rated voltage, the active power losses among different AC and DC transmission channels are different when transmitting the same power. An optimal transmission power distribution for different $\mathrm{AC}$ and DC transmission channels can effectively reduce the active power loss of the entire AC-DC interconnected transmission system, resulting in significant economic benefits.

The problem of optimal power distribution (OPD) for parallel transmission channels in an AC-DC interconnected power grid is the formulation of the optimal power transmission schedule of each $A C$ or DC transmission channel. This minimizes the sum of the active power loss of all the AC and DC 
transmission channels and satisfies the required total power exchange schedule constraints of the AC and DC tie-lines between provincial grids and the total transmission power security constraints on the AC transmission sections between provincial grids. It is always formulated as an optimal power flow (OPF) model for AC-DC systems. At present, the conventional OPF model for AC-DC systems is generally used in the optimal operation of AC-DC systems [5-14]. The optimal reactive power flow (ORPF) problem for AC-DC systems was studied in [5-9]. In [5], a heuristic genetic algorithm was used to solve the ORPF problem of a multi-terminal high voltage direct current (HVDC) transmission system. In [6], a quadratic ORPF model for an AC-DC hybrid power system was proposed, and it was solved with the predictor-corrector interior point method. Yu et al. [7] proposed an AC-DC ORPF model with generator capacity constraints, which was solved by a heuristic algorithm. In [8], a quadratic model of the power flow $(\mathrm{PF})$ and ORPF in AC-DC power systems was proposed, and the quadratic expression of a unified power flow controller was introduced in the model. In [9], a stability-constrained ORPF model for reactive power compensation and HVDC regulation was established, and several derivative-free algorithms were proposed. Moreover, the general OPF problem for AC-DC systems was studied in [10-12]. In [10], the adaptive simplified human learning optimization algorithm was used to solve the OPF problem of AC-DC systems. Aragüés-Peñalba et al. [11] proposed a method for solving the OPF problem of offshore wind farms with an HVDC connection, and they used an information gap decision theory to deal with the uncertainty of the wind power fluctuations. K1lıç et al. [12] used a genetic algorithm to solve the OPF problem of an AC-DC power grid. In addition, $[13,14]$ studied the multi-objective optimal operation problem of AC-DC systems. Cao et al. [13] established a multi-objective optimization model for AC-DC systems with wind farms with the goal of minimizing their operation cost, power loss, and pollutant emission. Li et al. [14] established a multi-objective AC-DC reactive power optimization model with the power loss and the voltage deviation as objective functions. Since the injected power balance constraints of each bus and the steady-state operation characteristic constraints of DC systems are non-convex constraints, the OPF model of an AC-DC system is a nonlinear non-convex programming model, which cannot guarantee the optimality and reliability of the solution results. Moreover, the above references did not consider the ratio adjustment of converter transformers in DC converter stations, i.e., the optimization model did not include the integer variables of the converter transformers' ratios. In addition, most of the existing research uses single-period OPD models, rarely considering the day-ahead multi-period OPD of AC-DC parallel transmission channels, which need to consider the regulating constraints of the DC lines' transmission power schedule during a day.

In the actual operation of AC-DC power grids, adjusting the transmission power of DC lines too frequently is harmful to electrical devices. Hence, the required transmission power schedule of a HVDC transmission channel is a stepped shape curve, and only a few transmission power states are in the curve. At present, the existing research usually adopts the multiple stages method to solve the problem. In other words, the DC lines' power adjustment constraint is not considered first, and the optimization model is solved to obtain the ideal OPD scheme of the AC-DC parallel transmission channels. Then the obtained transmission power schedule of the DC transmission channels executes the stepped shape processing with the curve fitting method. In this way, the obtained solution is often not the optimal solution of the problem. If the constraints of the stepped shape processing are directly added to the optimization model, then the OPD model of the AC-DC parallel transmission channels is a mixed-integer nonlinear non-convex programming (MINNP) model. The MINNP model is one of the most difficult problems in the field of mathematical programming, which belongs to the NP-hard problem classification. It is difficult to obtain the global optimal solution, and the solution efficiency is low. Therefore, to meet the requirement of practical engineering applications, it is necessary to propose a reasonable algorithm to obtain the optimal solution of the model reliably and improve the calculation efficiency. The second-order cone programming (SOCP) method is a convex optimization method that can obtain the global optimal solution and that has high calculation efficiency. Many researchers have studied the SOCP relaxation model of AC power flow equations, which is widely 
used in the fields of OPF [15,16], unit commitment [17], power system planning [18,19], etc. In terms of the OPF of AC-DC hybrid systems, Ding et al. [20] proposed an SOCP relaxation approach to solve the optimal power flow problem with hybrid voltage source converter-based high voltage direct current (VSC-HVDC) transmission and active distribution networks and with the radial AC branch power flow models relaxed. [21,22] applied the SOCP relaxation method to the optimal power flow models in VSC-type AC-DC grids. However, there is still no relevant research on applying the SOCP method to the dynamic OPF problem of AC-DC systems with the discrete variables of converter transformer ratio adjustment and the stepped shape processing of DC channels' transmission power schedule. Therefore, it is necessary to find a method for transforming the MINNP model of the OPD of AC-DC parallel transmission channels into a mixed-integer SOCP (MISOCP) model to obtain an efficient and reliable solution.

The main contributions of the current study are as follows: (1) A dynamic OPF model for day-ahead OPD of AC-DC parallel transmission channels is established. This model accounts for the power flow equation constraints in an AC-DC power grid, the discrete gear position adjustment of the converter transformer ratio, and the regulation constraints of the transmission power of the DC lines in actual operation, which can meet the requirement of formulating the day-ahead OPD schedule of AC-DC parallel transmission channels in actual operation. (2) The nonlinear objective function and the constraints are executed with convex relaxation using the cone relaxation transformation and the big $\mathrm{M}$ method, and the MINNP model is transformed into a MISOCP model, which can be solved reliably and efficiently to obtain the optimal solution.

The rest of the paper is organized as follows: Section 2 introduces the mathematical model for the OPD of AC-DC parallel transmission channels. Section 3 introduces the method for transforming the OPD model of AC-DC parallel transmission channels into a MISOCP model. Section 4 verifies the correctness of the proposed model and the algorithm with a case study of an actual AC-DC power grid. Section 5 is the conclusion.

\section{Mathematical Model for the OPD of AC-DC Parallel Transmission Channels}

\subsection{Objective Function}

The objective is to minimize the total electric energy loss of all the AC and DC transmission lines in all the AC-DC parallel transmission channels in a day, as shown in Formula (1):

$$
\min f=\sum_{t=1}^{T}\left(\sum_{l=1}^{N_{a c}} P_{L a, t}^{l}+\sum_{k=1}^{N_{d c}} P_{L d, t}^{k}\right) \Delta T
$$

where $T$ is the total number of time intervals in the OPD period, and $\Delta T$ is the time length of each time interval. If $\Delta T=15 \mathrm{~min}$, then 96 time intervals are included in a day and $T=96$; and if $\Delta T=1 \mathrm{~h}$, then $T=24$. $P_{L a, t}^{l}$ represents the active power loss of the $l$-th AC transmission line, and $P_{L d, t}^{k}$ represents the active power loss of the $k$-th DC transmission line. Subscript $t$ represents the variable of the time interval $t$, which is the same in the following formulae. $N_{a c}$ and $N_{d c}$ are the total number of AC and DC transmission lines included in all the AC-DC parallel transmission channels.

For the AC transmission lines, the active power loss can be formulated as Equation (2):

$$
P_{L a, t}^{l}=\left[\left(V_{a i, t}^{l}\right)^{2}+\left(V_{a j, t}^{l}\right)^{2}-2 V_{a i, t}^{l} V_{a j, t}^{l} \cos \left(\theta_{a i, t}^{l}-\theta_{a j, t}^{l}\right)\right] g_{a l}
$$

where $V_{a i, t}^{l}$ and $V_{a j, t}^{l}$ are the voltage amplitudes of the buses $i$ and $j$ connected to the $l$-th AC line, $\theta_{a i, t}^{l}$ and $\theta_{a j, t}^{l}$ are the voltage phase angles of the buses $i$ and $j$, and $g_{a l}$ is the conductance of the $l$-th AC line. For the DC transmission lines, the active power loss can be formulated as Equation (3):

$$
P_{L d, t}^{k}=n_{k}\left(I_{d, t}^{k}\right)^{2} R_{d k}
$$


where $n_{k}$ is the number of operating poles of the $k$-th DC line, and $I_{d, t}^{k}$ and $R_{d k}$ are the unipolar operating current and the unipolar resistance of the $k$-th DC line.

\subsection{Constraints}

(1) The total power exchange schedule constraints of each provincial network and the corresponding external networks can be described as

$$
-\varepsilon \% P_{s, t} \leq \sum_{l \in B_{p a}} P_{a, t}^{l}+\sum_{k \in B_{p d}} P_{d, t}^{k}-P_{s, t} \leq \varepsilon \% P_{s, t}
$$

where $P_{a, t}^{l}$ is the transmission power of the $l$-th AC tie-line, and $P_{d, t}^{k}$ is the transmission power of the $k$-th DC tie-line. If the rectifier side of the $k$-th DC tie-line is in the provincial network $s$, then $P_{d, t}^{k}=P_{d R, t^{\prime}}^{k}$ and if the inverter side of the $k$-th DC tie-line is in the provincial network $s$, then $P_{d, t}^{k}=P_{d I, t^{t}}^{k}$ and $P_{d R, t}^{k}$ and $P_{d I, t}^{k}$ are the DC power of the rectifier side and the inverter of the $k$-th DC tie-line, respectively. $P_{s, t}$ is the total power exchange value between the provincial network $s$ and the external networks. $B_{p a}$ and $B_{p d}$ are the set AC tie-lines and the DC tie-lines connected to the provincial network $s$ and the external networks, and $\varepsilon \%$ is the allowable deviation percentage of the power exchange schedule value between the provincial networks.

The transmission power of the AC lines is calculated as follows:

$$
P_{a, t}^{l}=V_{a i, t}^{l} V_{a j, t}^{l}\left[g_{a l} \cos \left(\theta_{a i, t}^{l}-\theta_{a j, t}^{l}\right)+b_{a l} \sin \left(\theta_{a i, t}^{l}-\theta_{a j, t}^{l}\right)\right]-\left(V_{a i, t}^{l}\right)^{2} g_{a l}
$$

where $b_{a l}$ is the susceptance of the $l$-th AC line.

The transmission power of the DC lines is calculated as follows:

$$
P_{d, t}^{k}=n_{k} V_{d, t}^{k} I_{d, t}^{k}
$$

where $V_{d, t}^{k}$ is the unipolar operating voltage of the $k$-th DC line.

(2) Security constraints for the AC transmission lines and the sections

The transmission power security constraints of the AC lines are as follows:

$$
-P_{l \max } \leq P_{a, t}^{l} \leq P_{l \max }
$$

where $P_{l \max }$ represents the maximum active power corresponding to the ampacity limit of the $l$-th AC line.

The transmission power security constraints of the AC transmission sections are as follows:

$$
P_{s a, \min } \leq \sum_{l \in B_{s}} P_{a, t}^{l} \leq P_{s a, \max }
$$

where $P_{s a, \min }$ and $P_{s a, \max }$ are the minimum and maximum transmission power of the $s$-th AC transmission section, respectively. $B_{s}$ is the set of AC lines included in the s-th AC transmission section.

(3) Operating constraints of the DC lines

The operational constraints of the DC transmission lines include the DC power equation, Equation (6), the basic equations of the converter station as Equations (9) and (10), and the DC network equation, Equation (11), as follows [23]:

$$
\begin{gathered}
V_{d, t}^{k}=k_{T, t}^{k} V_{i, t} \cos \theta_{d, t}^{k}-X_{c k} I_{d, t}^{k} \\
V_{d, t}^{k}=k_{\gamma} k_{T, t}^{k} V_{i, t} \cos \varphi_{k, t} \\
V_{d R, t}^{k}=V_{d I, t}^{k}+I_{d, t}^{k} R_{d k}
\end{gathered}
$$


where $V_{i, t}$ is the voltage of the AC commutation bus $i$ of the $k$-th DC line, $k_{T, t}^{k}$ is the ratio of the converter transformer of the $k$-th DC line, and $\theta_{d, t}^{k}$ is the control angle of the converter of the $k$-th DC line. $X_{c k}$ is the equivalent reactance of the converter transformer of the $k$-th DC line, and $k_{r}$ is a constant, which generally takes the value of 0.995. $\varphi_{k, t}$ is the power factor angle of the converter station of the $k$-th DC line, and $V_{d R, t}^{k}$ and $V_{d I, t}^{k}$ are the DC voltages of the rectifier side and the inverter side of the $\mathrm{k}$-th DC line.

(4) Power balance equations of the AC buses

The injected power balance equations of the AC bus $i$ are as follows:

$$
\left\{\begin{array}{l}
P_{G i, t}-P_{L i, t} \pm P_{d, t}^{k}=V_{i, t} \sum_{j=1}^{n} V_{j, t}\left(G_{i j} \cos \theta_{i j, t}+B_{i j} \sin \theta_{i j, t}\right) \\
Q_{G i, t}-Q_{L i, t}-P_{d, t}^{k} \tan \varphi_{k, t}=V_{i, t} \sum_{j=1}^{n} V_{j, t}\left(G_{i j} \sin \theta_{i j, t}-B_{i j} \cos \theta_{i j, t}\right)
\end{array}\right.
$$

where $P_{G i, t}$ and $Q_{G i, t}$ are the active and reactive power outputs of the generator at bus $i, P_{L i, t}$ and $Q_{L i, t}$ are the active and reactive power load at bus $i, P_{d, t}^{k}$ is the DC power of the $k$-th DC line connected to the AC node $i$, where the negative sign is taken for the rectifier station and the positive sign is taken for the inverter station, $G_{i j}$ and $B_{i j}$ are the real and imaginary parts of the $i$-th line and the $j$-th column element of the node admittance matrix, and $\theta_{i j, t}$ is the voltage phase angle difference between buses $i$ and $j$.

(5) Regulating limit of the DC lines' transmission power

In the practical operation of AC-DC interconnected power systems, too frequent regulation of the DC transmission power not only does harm to electrical equipment but also increases the work burden of operators. Therefore, the required transmission power schedules for DC transmission lines have step shaped curves including only a few transmission power states of step changes, that is, it is necessary to limit the maximum amount of the regulation of the DC lines' transmission power schedules during a day, and the minimum duration of each transmission power state should be limited. In addition, to reduce the impact on the regional AC network near a DC converter station, the allowable power variation of each regulation should not be too large. Therefore, the regulating limits for the transmission power of DC lines are as follows:

$$
\left\{\begin{array}{l}
P_{d, t}^{k}=P_{d, t-1}^{k}+u_{t}^{k} \Delta P_{d, t}^{k} \\
u_{t}^{k} \Delta P_{k \min } \leq \Delta P_{d, t}^{k} \leq u_{t}^{k} \Delta P_{k \max } \\
\sum_{t=1}^{T} u_{t}^{k} \leq C_{k}^{a d j} \\
u_{t}^{k}+\sum_{w=1}^{T_{w}} u_{t+w}^{k} \leq 1
\end{array}\right.
$$

where $\Delta P_{d, t}^{k}$ is the regulating power of the $k$-th DC line, $\Delta P_{k \max }$ and $\Delta P_{k \min }$ are the maximum and minimum power of each regulation of the $k$-th DC line, $u_{t}^{k}$ is a $0 / 1$ integer variable that indicates whether the transmission power of the $k$-th DC line is regulated, 0 represents the fact that the transmission power at time interval $t$ is maintained the same value as that of the previous time interval $t-1$, and 1 represents the fact that the transmission power at time interval $t$ will increase or decrease based on the value of the previous time interval $t-1$. $C_{k}^{a d j}$ is the allowable amount of power regulation for the $k$-th DC transmission line during a day. $T_{w}$ represents the fact that the minimum duration of each transmission power state has $\left(T_{w}+1\right)$ time intervals.

(6) Upper and lower limits of the variables

The upper and lower limits of the variables include the limits of the DC line transmission power, the DC voltage, the DC line transmission current, the converter transformer ratio, the converter control angle, the voltages of the $\mathrm{AC}$ buses, and the active and reactive power output of the generator at the swing bus, as follows:

$$
x_{\min } \leq x \leq x_{\max }
$$

where $x=\left\{P_{d, t^{\prime}}^{k} V_{d, t^{\prime}}^{k} I_{d, t^{\prime}}^{k} k_{T, t^{\prime}}^{k} \theta_{d, t^{\prime}}^{k} V_{i, t}, P_{G s w, t}, Q_{G s w, t}\right\} . P_{G s w, t}$ and $Q_{G s w, t}$ are the active and reactive power output of the generator at the swing bus. 
In the above OPD model of the AC-DC parallel transmission channels for Equations (1)-(14), Equations (2) and (3) are used to calculate the active power loss of the AC and DC lines in the objective function, Equations (5) and (6) are used to calcute the transmission power of the AC and DC lines, Equations (9) and (10) are used for the operating characteristics of the converters, and the injected power balance equation is Equation (12) of the AC buses in the constraints. All the equations include the nonlinear items of the triangular functions or quadratic functions. Moreover, $k_{T, t}^{k}$ and $u_{t}^{k}$ are discrete decision variables. Therefore, the optimization model is an MINNP model, which is an NP-hard problem, and it is very difficult to obtain the optimal solution.

\section{MISOCP Algorithm for the OPD of the AC-DC Parallel Transmission Channels}

For the MINNP problem of the OPD of AC-DC parallel transmission channels in actual large-scale power systems, the scale of the optimization model to be solved is very large. If the model is solved by a common MINLP solver such as SBB in the mature commercial optimization software GAMS, then the calculation efficiency will be very low, and the optimal solution of the optimization problem generally cannot be obtained. Therefore, in this research, a convex relaxation was applied to transform the nonlinear objective function and constraints into convex forms with second-order cone (SOC) relaxation and the big M method. Then the OPD model of AC-DC parallel transmission channels was transformed into a MISOCP model whose computational complexity was effectively reduced.

\subsection{Convex Relaxation of DC Line Constraints}

In the actual operation of AC-DC power systems, since the AC bus voltage is generally close to its rated voltage, the voltage of the AC commutation bus $i$ of the $k$-th DC line can be approximately equal to 1.0 p.u.; then Equation (9) of the converters' operating characteristics can be approximately simplified to $V_{d, t}^{k}=k_{T, t}^{k} \cos \theta_{d, t}^{k}-X_{c k} I_{d, t}^{k}$. After the converter transformer ratio is discretized as Equation (15), the DC voltage can be further described as Equation (16):

$$
\begin{gathered}
k_{T, t}^{k}=\sum_{i=1}^{N_{r}} k_{T i, t}^{k} T_{i k} \\
V_{d, t}^{k}=\sum_{i=1}^{N_{r}} k_{T i, t}^{k} T_{i k} \cos \theta_{d, t}^{k}-X_{c k} k_{d, t}^{k}
\end{gathered}
$$

where $k_{T i, t}^{k}$ indicates the operating status whether the tap of the converter transformer of the $k$-th DC line is operating in the $i$-th regulating gear, which is a binary discrete variable $0 / 1$ that must satisfy $\sum_{i=1}^{N_{r}} k_{T i, t}^{k}=1$. $T_{i k}$ is the ratio value corresponding to the $i$-th voltage regulating gear of the converter transformer, and $N_{r}$ is the number of voltage-regulating gears of the converter transformer.

Considering $\cos \theta_{d, t}^{k}$ as a whole continuous variable, then $k_{T i, t}^{k} \cos \theta_{d, t}^{k}$ is the product of a discrete variable and a continuous variable, which can be transformed into linear constraints with the big $\mathrm{M}$ method. A continuous variable $H_{i, t}^{k}$ that is equal to $k_{T i, t}^{k} \cos \theta_{d, t}^{k}$ is introduced, and $H_{i, t}^{k}=k_{T i, t}^{k} \cos \theta_{d, t}^{k}$ can be reformulated as linear inequalities using the $\operatorname{big} M$ method as follows:

$$
\left\{\begin{array}{l}
-M \cdot k_{T i, t}^{k} \leq H_{i, t}^{k} \leq M \cdot k_{T i, t}^{k} \\
-M\left(1-k_{T i, t}^{k}\right)+\cos \theta_{d, t}^{k} \leq H_{i, t}^{k} \leq M\left(1-k_{T i, t}^{k}\right)+\cos \theta_{d, t}^{k}
\end{array}\right.
$$

where $M$ is a constant with a large value.

Thus, Equation (16) can be further transformed into linear constraints as follows:

$$
\left\{\begin{array}{l}
V_{d, t}^{k}=\sum_{i=1}^{N_{r}}\left(T_{i k} H_{i, t}^{k}\right)-X_{c k} I_{d, t}^{k} \\
-M \cdot k_{T i, t}^{k} \leq H_{i, t}^{k} \leq M \cdot k_{T i, t}^{k} \\
-M\left(1-k_{T i, t}^{k}\right)+\cos \theta_{d, t}^{k} \leq H_{i, t}^{k} \leq M\left(1-k_{T i, t}^{k}\right)+\cos \theta_{d, t}^{k} \\
\sum_{i=1}^{N_{r}} k_{T i, t}^{k}=1
\end{array}\right.
$$


For the transmission power equation of DC lines, Equation (6), since the constant DC voltage control is usually adopted at the rectifier side in the actual operation, the DC voltage at the rectifier side can be fixed to its rated voltage; that is, $V_{d R, t}^{k}=V_{d R N}^{k}=$ const. Then the transmission power at the rectifier side $P_{d R, t}^{k}=n_{k} V_{d R, t}^{k} I_{d, t}^{k}$ can be converted into a linear equality constraint as $P_{d R, t}^{k}=n_{k} V_{d R N}^{k} I_{d, t}^{k}$. The conversion of the transmission power at the inverter side into the convex constraint is as follows:

$$
\begin{aligned}
& P_{d I, t}^{k}=n_{k} V_{d I, t}^{k} I_{d, t}^{k}=n_{k}\left(V_{d R, t}^{k}-I_{d, t}^{k} R_{d k}\right) I_{d, t}^{k}=n_{k}\left(V_{d R N}^{k}-I_{d, t}^{k} R_{d k}\right) I_{d, t}^{k} \\
& \Rightarrow-\frac{P_{d l, t}^{k}}{n_{k} R_{d k}}+\left(\frac{V_{d R N}^{k}}{2 R_{d k}}\right)^{2}=\left(I_{d, t}^{k}-\frac{V_{d R N}^{k}}{2 R_{d k}}\right) .
\end{aligned}
$$

This equation is a quadratic equality constraint on the DC current $I_{d, t^{\prime}}^{k}$ which can be transformed into a convex quadratic inequality by SOC relaxation as follows [24]:

$$
\left\|\left[2\left(I_{d, t}^{k}-\frac{V_{d R N}^{k}}{2 R_{d k}}\right),-\frac{P_{d I, t}^{k}}{n_{k} R_{d k}}+\left(\frac{V_{d R N}^{k}}{2 R_{d k}}\right)^{2}-1\right]\right\|_{2}^{\mathrm{T}} \leq-\frac{P_{d I, t}^{k}}{n_{k} R_{d k}}+\left(\frac{V_{d R N}^{k}}{2 R_{d k}}\right)^{2}+1 .
$$

Equation (10) is an expression for calculating the power factor of the complex power absorbed by the converter station from the AC system, which is a nonlinear equality including the cosine function of $\varphi_{k, t}$. The variable of the reactive power $Q_{d, t}^{k}$ can be introduced to replace the power factor angle $\varphi_{k, t}$ for convex relaxation processing. Assigning $Q_{d, t}^{k}$ to be the reactive power absorbed by the converter station of the $k$-th DC line from the AC system, while neglecting the active power loss of the converter, it can be expressed as follows:

$$
P_{d, t}^{k}+j Q_{d, t}^{k}=k_{\gamma} k_{T, t}^{k} V_{i, t} I_{d, t}^{k}\left(\cos \varphi_{k, t}+j \sin \varphi_{k, t}\right)
$$

Letting $S_{d, t}^{k}=k_{\gamma} k_{T, t}^{k} V_{i, t} I_{d, t}^{k} \approx k_{\gamma} k_{T, t_{d, t^{\prime}}^{k}}^{k} I^{k}$ then $\left(P_{d, t}^{k}\right)^{2}+\left(Q_{d, t}^{k}\right)^{2}=\left(S_{d, t}^{k}\right)^{2}$ can be established, which can be transformed into a convex quadratic inequality via SOC relaxation as follows:

$$
\left\|\left(P_{d, t^{\prime}}^{k} Q_{d, t}^{k}\right)^{\mathrm{T}}\right\|_{2} \leq S_{d, t}^{k}
$$

Moreover, for the quadratic equality $S_{d, t}^{k}=k_{\gamma} k_{T, t}^{k} I_{d, t}^{k}=k_{\gamma} \sum_{i=1}^{N_{r}} k_{T i, t}^{k} T_{i k} I_{d, t^{\prime}}^{k}$ by introducing a continuous variable $W_{i, t}^{k}$ that is equal to $k_{T i, t}^{k} I_{d, t}^{k}$ and applying the big M method, the formula can be further transformed into the linear constraints as follows:

$$
\left\{\begin{array}{l}
S_{d, t}^{k}=k_{\gamma} \sum_{i=1}^{N_{r}} T_{i k} W_{i, t}^{k} \\
-M \cdot k_{T i, t}^{k} \leq W_{i, t}^{k} \leq M \cdot k_{T i, t}^{k} \\
-M\left(1-k_{T i, t}^{k}\right)+I_{d, t}^{k} \leq W_{i, t}^{k} \leq M\left(1-k_{T i, t}^{k}\right)+I_{d, t}^{k}
\end{array}\right.
$$

It can be seen that in Equations (21)-(23), $\varphi_{k, t}$ is replaced by introducing $Q_{d, t^{\prime}}^{k}$ which can eliminate the nonlinear constraint of the cosine function of $\varphi_{k, t}$ in Equation (10). At the same time, $P_{d, t}^{k} \tan \varphi_{k, t}$ in the second formula of Equation (12) also needs to be replaced by $Q_{d, t}^{k}$.

\subsection{SOC Relaxation of the Injected Power Balance Equations of the AC Buses}

The following variables are introduced as intermediate variables:

$$
\left\{\begin{array}{c}
Y_{i j, t}=V_{i, t} V_{j, t} \cos \theta_{i j, t} \\
Z_{i j, t}=V_{i, t} V_{j, t} \sin \theta_{i j, t} \\
X_{i, t}=V_{i, t}^{2}
\end{array}\right.
$$


The introduced intermediate variables must satisfy the following constraints:

$$
\begin{gathered}
Y_{i j, t}=Y_{j i, t} \\
Z_{i j, t}=-Z_{j i, t} \\
Y_{i j, t}^{2}+Z_{i j, t}^{2}=X_{i, t} X_{j, t}
\end{gathered}
$$

Formula (27) can be converted into a convex quadratic inequality via the SOC relaxation as follows [24]:

$$
\left\|\left(2 Y_{i j, t}, 2 Z_{i j, t}, X_{i, t}-X_{j, t}\right)^{\mathrm{T}}\right\|_{2} \leq X_{i, t}+X_{j, t} .
$$

Then the injected power balance equation of the AC buses, Equation (12), can be transformed into the linear constraints as follows:

$$
\left\{\begin{array}{c}
P_{G i, t}-P_{L i, t} \pm P_{d c, t}^{k}=G_{i i} X_{i, t}+\sum_{j=1, j \neq i}^{n}\left(G_{i j} Y_{i j, t}+B_{i j} Z_{i j, t}\right) \\
Q_{G i, t}-Q_{L i, t}-Q_{d c, t}^{k}=-B_{i i} X_{i, t}-\sum_{j=1, j \neq i}^{n}\left(B_{i j} Y_{i, t}-G_{i j} Z_{i j, t}\right)
\end{array} .\right.
$$

The upper and lower limits of the AC buses voltage can then be rewritten as follows:

$$
V_{i, \min }^{2} \leq X_{i, t} \leq V_{i, \max }^{2}
$$

\subsection{Linearization of the Active Power Loss and the Transmission Power of the AC Lines}

It can be determined from the variable substitution in Section 3.2 that the active power loss equation, Equation (2), of the AC lines in the objective function can be converted into a linear expression as

$$
P_{L a, t}^{l}=\left(X_{a i, t}+X_{a j, t}-2 Y_{a i j . t}\right) g_{a l}
$$

Moreover, the transmission power equation, Equation (5) of the AC lines, can be converted into a linear expression as

$$
P_{a, t}^{l}=\left(Y_{a i j . t} g_{a l}+Z_{a i j . t} b_{a l}\right)-X_{a i, t} g_{a l}
$$

\subsection{Linearization of the Step Shape Constraint of the Transmission Power Schedules of the DC Lines}

Assuming that $\omega_{t}^{k}=u_{t}^{k} \Delta P_{d c, t^{\prime}}^{k}$ the constraint from Equation (13) of the DC transmission power regulating limit is linearized with the big $\mathrm{M}$ method as follows:

$$
\left\{\begin{array}{l}
P_{d, t}^{k}=P_{d, t-1}^{k}+\omega_{t}^{k} \\
-M \cdot u_{t}^{k} \leq \omega_{t}^{k} \leq M \cdot u_{t}^{k} \\
-M\left(1-u_{t}^{k}\right)+\Delta P_{k \min } \leq \omega_{t}^{k} \leq M\left(1-u_{t}^{k}\right)+\Delta P_{k \max } \\
\sum_{t=1}^{T} u_{t}^{k} \leq C_{k}^{a d j} \\
u_{t}^{k}+\sum_{w=1}^{T_{w}} u_{t+w}^{k} \leq 1
\end{array}\right.
$$

In summary, after the convex relaxation of the nonlinear items in the optimization model, the OPD model of AC-DC parallel transmission channels is transformed into a MISOCP model with convex quadratic objective function and linear or SOC constraints. In other words, Equations (1), (31), and (3) 
constitute the objective function, and Equations (4), (7), (8), (11), (12), (18), (20), (22), (23), (25), (26), (28)-(30), (32), and (33) constitute the constraints as follows:

$$
\begin{aligned}
& \min f=\sum_{t=1}^{T}\left[\sum_{l=1}^{N_{a c}}\left(X_{a i, t}+X_{a j, t}-2 Y_{a i j . t}\right) g_{a l}+\sum_{k=1}^{N_{d c}} n_{k}\left(I_{d, t}^{k}\right)^{2} R_{d k}\right] \Delta T \text { s.t. } \\
& \left\{\begin{array}{l}
V_{d, t}^{k}=\sum_{i=1}^{N_{r}}\left(T_{i k} H_{i, t}^{k}\right)-X_{c k} I_{d, t}^{k} \\
-M \cdot k_{T i, t}^{k} \leq H_{i, t}^{k} \leq M \cdot k_{T i, t}^{k} \\
-M\left(1-k_{T i, t}^{k}\right)+\cos \theta_{d, t}^{k} \leq H_{i, t}^{k} \leq M\left(1-k_{T i, t}^{k}\right)+\cos \theta_{d, t}^{k} \\
\sum_{i=1}^{N_{r}} k_{T i, t}^{k}=1 \\
V_{d I, t}^{k}=V_{d R N}^{k}-I_{d, t}^{k} R_{d k} \\
P_{d R, t}^{k}=n_{k} V_{d R N}^{k} I_{d, t}^{k} \\
\left\|\left[2\left(I_{d, t}^{k}-\frac{V_{d R N}^{k}}{2 R_{d k}}\right),-\frac{P_{d, t}^{k}}{n_{k} R_{d k}}+\left(\frac{V_{d R N}^{k}}{2 R_{d k}}\right)^{2}-1\right]\right\|_{2} \leq-\frac{P_{d, t}^{k}}{n_{k} R_{d k}}+\left(\frac{V_{d R N}^{k}}{2 R_{d k}}\right)^{2}+1 \\
\left\|\left(P_{d, t^{\prime}}^{k} Q_{d, t}^{k}\right)^{\mathrm{T}}\right\|_{2} \leq S_{d, t}^{k} \\
S_{d, t}^{k}=k_{\gamma} \sum_{i=1}^{N_{r}} T_{i k} W_{i, t}^{k} \\
-M \cdot k_{T i, t}^{k} \leq W_{i, t}^{k} \leq M \cdot k_{T i, t}^{k} \\
-M\left(1-k_{T i, t}^{k}\right)+I_{d, t}^{k} \leq W_{i, t}^{k} \leq M\left(1-k_{T i, t}^{k}\right)+I_{d, t}^{k} \\
P_{d, t}^{k}=P_{d, t-1}^{k}+\omega_{t}^{k} \\
-M \cdot u_{t}^{k} \leq \omega_{t}^{k} \leq M \cdot u_{t}^{k} \\
-M\left(1-u_{t}^{k}\right)+\Delta P_{k \min } \leq \omega_{t}^{k} \leq M\left(1-u_{t}^{k}\right)+\Delta P_{k \max } \\
\sum_{t=1}^{T} u_{t}^{k} \leq C_{k}^{a d j} \\
u_{t}^{k}+\sum_{w w=1}^{T_{w}} u_{t+w}^{k} \leq 1 \\
P_{G i, t}-P_{L i, t} \pm P_{d, t}^{k}=G_{i i} X_{i, t}+\sum_{j=1, j \neq i}^{n}\left(G_{i j} Y_{i j, t}+B_{i j} Z_{i j, t}\right) \\
Q_{G i, t}-Q_{L i, t}-Q_{d, t}^{k}=-B_{i i} X_{i, t}-\sum_{j=1, j \neq i}^{n}\left(B_{i j} Y_{i, t}-G_{i j} Z_{i j, t}\right) \\
V_{i, \min }^{2} \leq X_{i, t} \leq V_{i, \max }^{2} \\
\left\|\left(2 Y_{i j, t} 2 Z_{i j, t} X_{i, t}-X_{j, t}\right)^{\mathrm{T}}\right\|_{2} \leq X_{i, t}+X_{j, t} \\
Y_{i j, t}=Y_{j i, t}, Z_{i j, t}=-Z_{j i, t} \\
-\varepsilon \% P_{s, t} \leq \sum_{l \in B_{p a}} P_{a, t}^{l}+\sum_{k \in B_{p d}} P_{d, t}^{k}-P_{s, t} \leq \varepsilon \% P_{s, t} \\
-P_{l \max } \leq P_{a, t}^{l} \leq P_{l \max } \\
P_{s a, \min } \leq \sum_{l \in B_{s}} P_{a, t}^{l} \leq P_{s a, \max } \\
P_{a, t}^{l}=\left(Y_{a i j . t} g_{a l}+Z_{a i j . t} b_{a l}\right)-X_{a i, t} g_{a l} \\
\end{array}\right.
\end{aligned}
$$

The above MISOCP model for the OPD of AC-DC parallel transmission channels can be solved reliably and efficiently by the GUROBI solver in the mature commercial optimization software GAMS [25]. The detailed information about inputs/outputs of the proposed MISOCP algorithm for the OPD of AC-DC parallel transmission channels is shown in Figure 1. 


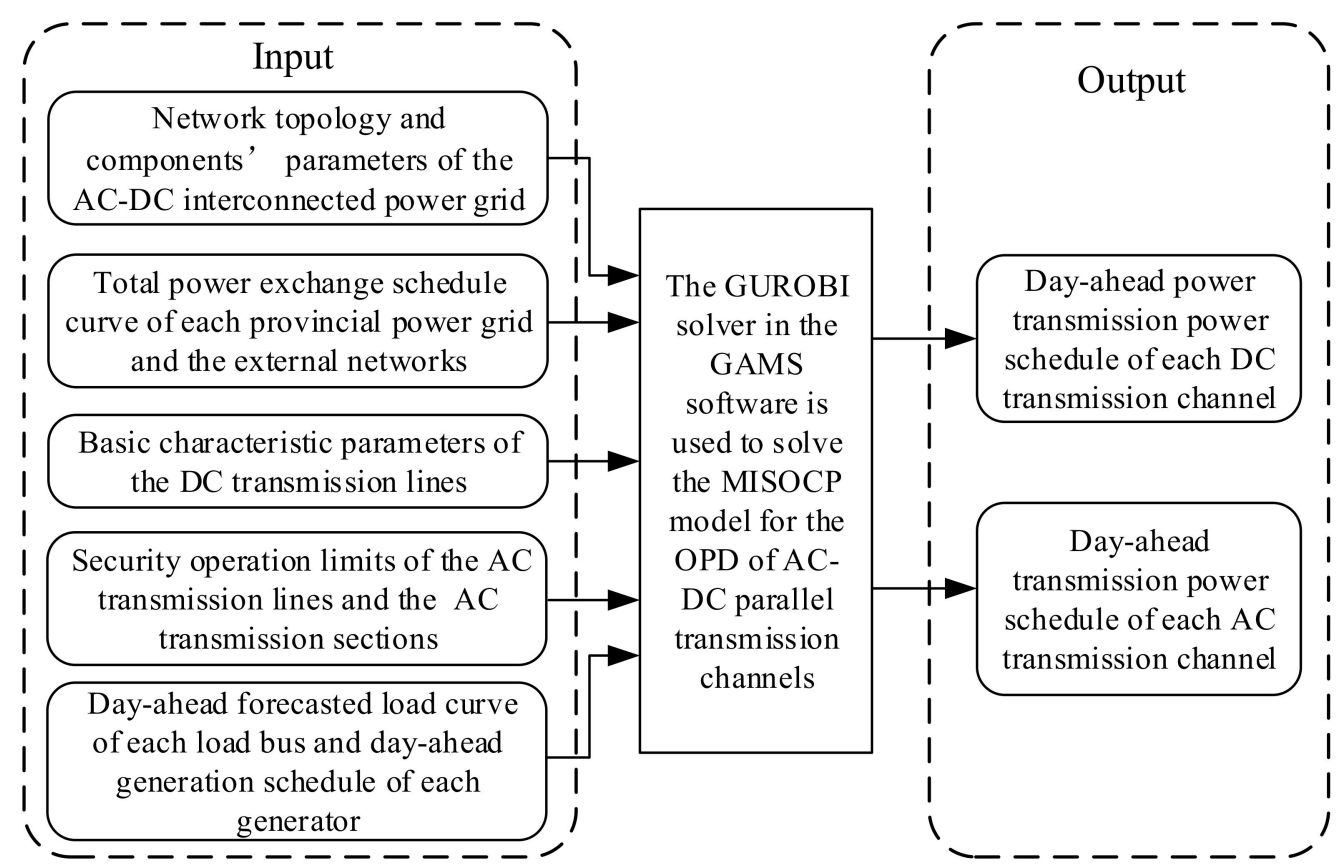

Figure 1. Detailed information about inputs/outputs of the proposed MISOCP algorithm for the OPD of AC-DC parallel transmission channels.

\section{Case Study}

\subsection{Structure and Parameters of the AC-DC Interconnected Power Grid}

A large-scale AC-DC interconnected power grid, i.e., the China Southern Power Grid, was taken as an example. The date of 1 August 2017 was chosen to analyze the computation performance of the proposed MISOCP method for the OPD model of AC-DC parallel transmission channels. The main grid structure of the AC-DC interconnected power grid is shown in Figure 2. The AC-DC parallel transmission channels included eight AC transmission channels and eight DC transmission channels (the Niu-Cong double-circuit DC transmission lines on the same tower were merged into one DC transmission line in this study). There were five DC transmission lines in the Yunnan power transmission section (including Luxi back-to-back DC), two DC transmission lines and four AC transmission lines in the Guizhou power transmission section, and six DC transmission lines and eight AC transmission lines in the Guangdong power transmission section. The main grid had 166 buses and 210 branches. Figure 3 shows the total power exchange schedule curve of the three provincial power transmission sections in one day. Table 1 shows the basic characteristic parameters of eight DC transmission lines. Table 2 shows the security limits of the key AC transmission sections between provinces. In Equation (13), $C_{k}^{a d j}$ took a value of 10, the minimum duration of the DC power transmission was set to $1 \mathrm{~h}$, that is, $T_{w}=3$ corresponded to $\Delta T=15 \mathrm{~min}$, and $\Delta P_{k \max }$ and $\Delta P_{k \min }$ were set to $10 \%$ and $-10 \%$ of the rated power of each DC line. The $M$ values in the constraints (17), (23), and (33) are set to be five times the maximum of the corresponding continual variables $\cos \theta_{d, t^{\prime}}^{k} I_{d, t^{\prime}}^{k}$, and $\Delta P_{\max }^{k}$. The computer used had an Intel(R) Xeon(R) CPU E3-1270 3.60 GHz, 32 GB memory, and the GAMS software version used for the optimization calculation was GAMS win64 24.5.3. 


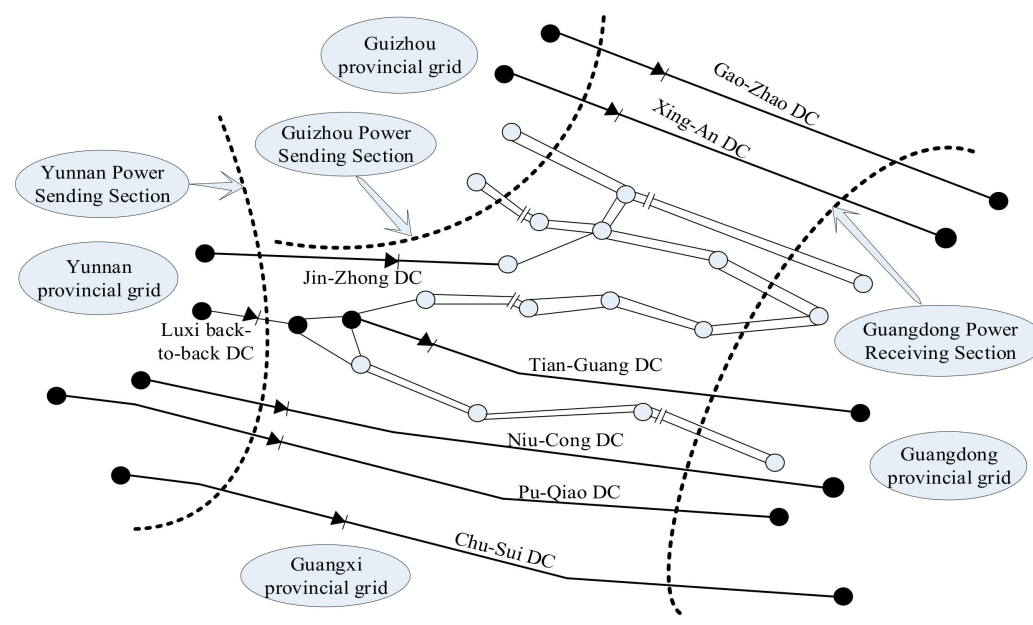

Figure 2. Main grid structure of the AC-DC interconnected power grid.

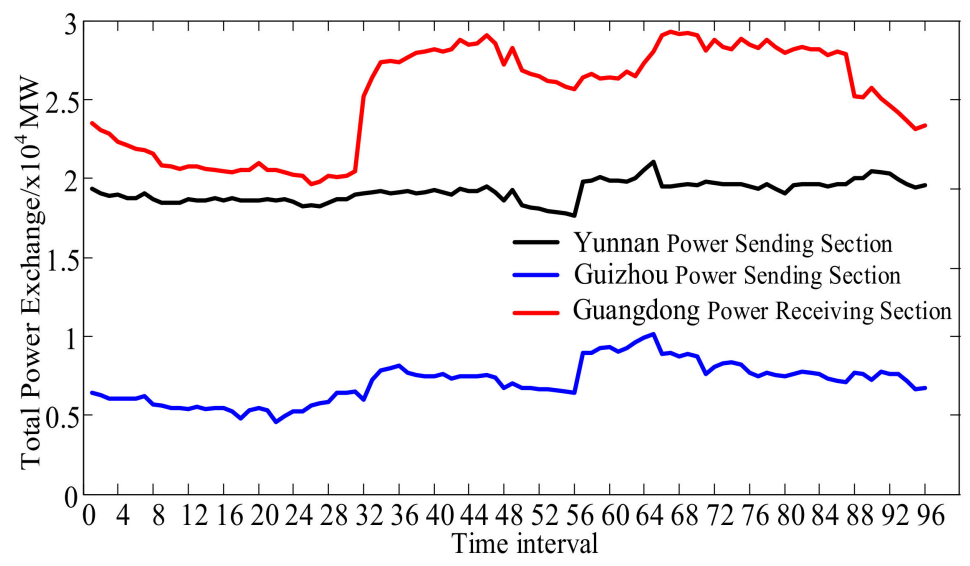

Figure 3. Total power exchange schedule curve of each provincial power grid and the external networks.

Table 1. Basic characteristic parameters of the DC transmission lines.

\begin{tabular}{ccccc}
\hline DC Line & Operating Pole Number & Line Resistance/ $\mathbf{\Omega}$ & Rated Voltage/kV & Rated Power/MW \\
\hline Tian-Guang DC & 2 & 22.16 & \pm 500 & 1800 \\
Gao-Zhao DC & 2 & 10.00 & \pm 500 & 3000 \\
Xing-An DC & 2 & 12.15 & \pm 500 & 3000 \\
Chu-Sui DC & 2 & 11.17 & \pm 800 & 5000 \\
Pu-Qiao DC & 2 & 11.17 & \pm 800 & 5000 \\
Niu-Cong DC & 4 & 9.664 & \pm 500 & 6400 \\
Jin-Zhong DC & 2 & 9.17 & \pm 500 & 3200 \\
Luxi back-to-back DC & 2 & 0.03 & \pm 500 & 2000 \\
\hline
\end{tabular}

Table 2. Security operation limits of key AC transmission sections.

\begin{tabular}{cc}
\hline Section Name & Security Limit of Transmission Power/MW \\
\hline Guizhou sending section & -300 to 3500 \\
Guangdong receiving section & $0-8500$ \\
\hline
\end{tabular}

\subsection{Calculation Results and Discussion}

The MISOCP model for OPD of AC-DC parallel transmission channels was solved to obtain the OPD scheme. The total losses of the AC and DC parallel transmission channels in each time interval of the entire day were compared with the total losses before optimization, as shown in Figure 4 . It can be seen that the total loss of AC and DC parallel transmission channels in each time interval after 
optimization decreased compared with the total losses before optimization. The electric energy loss of the transmission channels in a day was $44,636 \mathrm{MWh}$ before optimization, and the electric energy loss was 38,473 MWh after optimization. The electric energy loss after optimization was $13.8 \%$ lower than that before optimization. It was assumed that the price of electricity was $¥ 300 / \mathrm{MWh}$, so the saved electricity energy cost was $¥ 1,849,000$ on this day, resulting in obvious economic benefits. Therefore, the proposed MISOCP method for OPD of AC-DC parallel transmission channels could effectively reduce the network losses and improve the economic benefits in the AC-DC system operation.

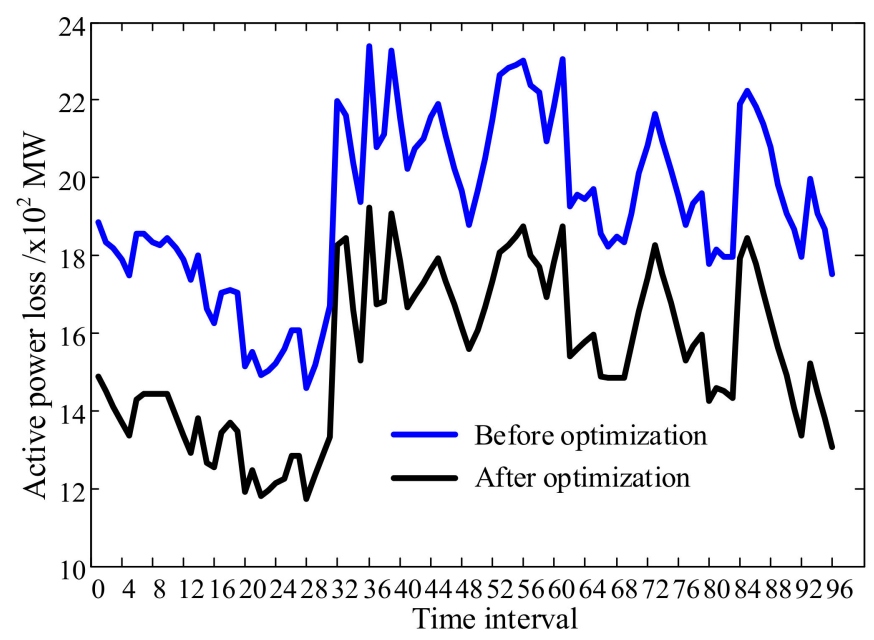

Figure 4. Total active power losses of AC and DC transmission channels before and after optimization.

The comparison of the transmission power schedule of each DC channel before and after optimization is shown in Figure 5, in which the transmission power is the DC power at the rectifier side. It can be seen that for the six DC channels connected to the Guangdong provincial gird and for the DC channels with relatively large line resistance, such as Tian-Guang DC and Xing-An DC, the power loss was higher than other DC channels when transmitting the same power, and their transmission power amounts after optimization were lower than the original values. For the two ultra high voltage direct current (UHVDC) lines of Chu-Sui DC and Pu-Qiao DC and another HVDC line Niu-Cong DC with relatively small line resistance, their transmission power amounts after optimization were bigger than the original values. These transmission power regulations were beneficial to reducing the active power loss of the DC lines. Similarly, for the two DC channels from the Yunnan province to the Guangxi province, the Luxi back-to-back DC line was very short, and the resistance was very small. Therefore, its transmission power after optimization increased compared with the power before optimization, and the transmission power of the Jin-Zhong DC was reduced compared with the power before optimization, which was also beneficial to reducing the active power loss of the DC lines. In addition, it can be seen from Figure 5 that the transmission power schedule of each DC channel after optimization could satisfy the requirements of no more than 10 transmission power regulation times in one day, and the minimum duration of each transmission power state was more than $1 \mathrm{~h}$. 


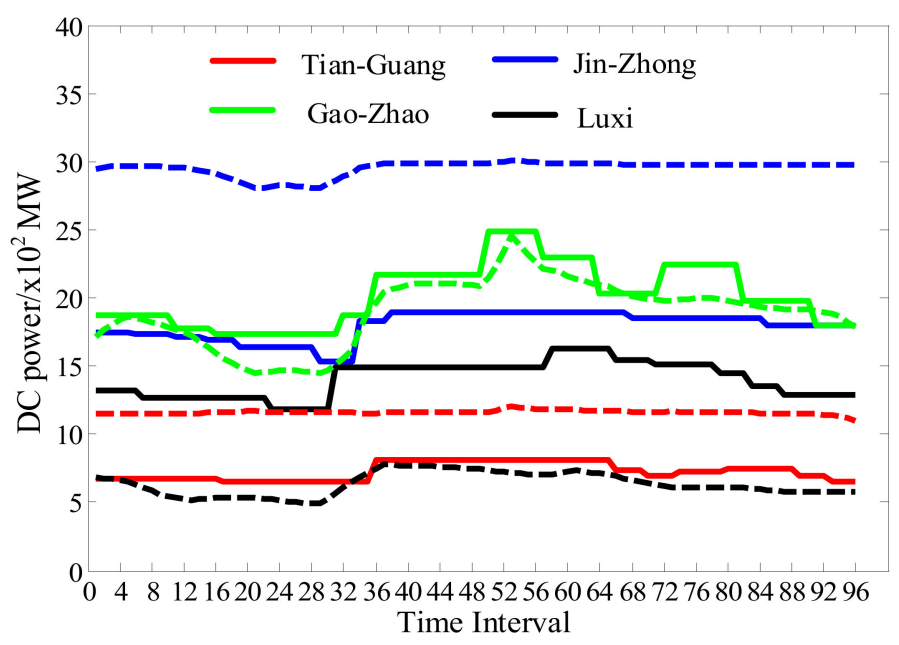

(a)

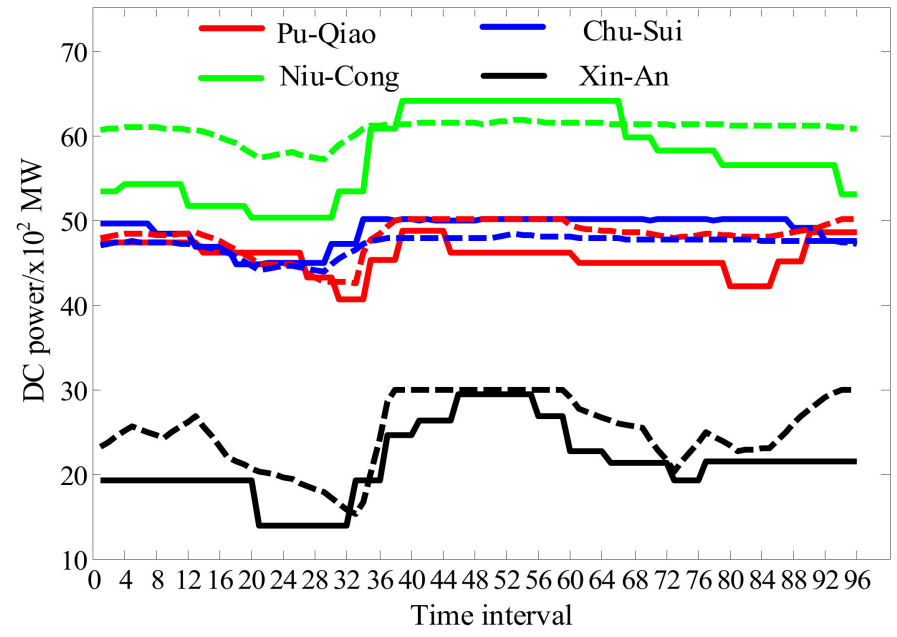

(b)

Figure 5. Comparison of the DC channel power before and after optimization (the dashed line represents the result before optimization, and the solid line represents the result of optimization). (a) Four DC channels; (b) Other four DC channels.

The optimal solution and the computational time of the MISOCP model proposed in this paper were compared with those of the MINNP model before convex relaxation with Equations (1)-(14), as shown in Table 3. The MINNP model before convex relaxation was solved by the SBB solver. For the single time interval OPD of the AC-DC parallel transmission channels, the constraints including the variables for multiple time intervals, i.e., the third and fourth formulae in Equation (13), were removed in the optimization. From the optimization results of three different time intervals, it can be seen that the computational time for solving the MISOCP model was significantly reduced compared with the computational time for solving the MINNP model. Moreover, the objective function of the MISOCP algorithm was smaller than that of the MINNP algorithm, indicating that the solution obtained by the MISOCP algorithm was better. Since the computational scale of the optimization model increased for the optimization of the 24 time intervals in one day $(\Delta T=1 \mathrm{~h}$, and $T=24)$ and the number of integer variables became too large, the solution of the MINNP model could not obtain an optimal integer solution, and only a feasible solution was obtained. When the computational scale was extended to the 96 time intervals in one day, the MINNP model could not converge, while the MISOCP could still obtain the optimal integer solution. In addition, for the optimization of 24 time intervals in one day, if the values of the integer variables of the optimal solution obtained by GUROBI for solving the MISOCP model were taken as the given variables and substituted into the MINNP model before 
convex relaxation, the objective function value obtained by the SBB solver was $38,667 \mathrm{MWh}$. This value was very close to the objective function value for the MISOCP model of 38,695 MWh, indicating that the proposed MISOCP model had high accuracy for the original MINNP model before convex relaxation.

Table 3. Comparison of the results of different optimization models with different solvers.

\begin{tabular}{|c|c|c|c|c|c|c|}
\hline \multirow[b]{2}{*}{$\begin{array}{l}\text { Optimization } \\
\text { Time Intervals }\end{array}$} & \multicolumn{3}{|c|}{ MISOCP (GUROBI) } & \multicolumn{3}{|c|}{ MINLP (SBB) } \\
\hline & $\begin{array}{c}\text { Objective } \\
\text { Function/ } \\
\text { MWh }\end{array}$ & CPU Time/s & $\begin{array}{l}\text { Solution } \\
\text { State }\end{array}$ & $\begin{array}{c}\text { Objective } \\
\text { Function/ } \\
\text { MWh }\end{array}$ & CPU Time/s & Solution State \\
\hline $\begin{array}{l}\text { Single time } \\
\text { interval }\end{array}$ & 1244 & 1.5 & $\begin{array}{l}\text { Integer } \\
\text { solution }\end{array}$ & 1253 & 3.4 & Integer solution \\
\hline 24 time intervals & 38,695 & 995 & $\begin{array}{l}\text { Integer } \\
\text { solution }\end{array}$ & 39,555 & 3253 & $\begin{array}{l}\text { Integer cannot } \\
\text { converge }\end{array}$ \\
\hline 96 time intervals & 38,473 & 5549 & $\begin{array}{l}\text { Integer } \\
\text { solution }\end{array}$ & - & - & Non-convergent \\
\hline
\end{tabular}

For the optimal solutions of the single time interval, 24 time intervals, and 96 time intervals OPD models of the AC-DC parallel transmission channels and for Equations (20), (22), and (28), which were relaxed into quadratic inequality constraints, the percentage of deviation between the two sides of the inequalities is shown in Table 4. It can be seen that after the SOC relaxation transformation, the deviations on both sides of the three quadratic inequality constraints were very small, and the maximum relative deviations were all below $10^{-4}$, very close to 0 . It can be seen that the proposed MISOCP model had high calculation accuracy, and the obtained solution was very close to satisfying the original quadratic equality constraints. Therefore, the solution of the proposed MISOCP algorithm had high precision for the original MINNP algorithm.

Table 4. Percentage deviation of the quadratic equality constraints relaxed as inequalities in the solution results.

\begin{tabular}{cccc}
\hline \multirow{2}{*}{$\begin{array}{c}\text { Quadratic Equality Constraints } \\
\text { Relaxed as Inequalities }\end{array}$} & $\begin{array}{c}\text { Single Time Interval } \\
\text { Optimization }\end{array}$ & $\begin{array}{c}\text { 24 Time Intervals } \\
\text { Optimization }\end{array}$ & $\begin{array}{c}\mathbf{9 6} \text { Time Intervals } \\
\text { Optimization }\end{array}$ \\
\cline { 2 - 4 }$\left(I_{d, t}^{k}-\frac{V_{d R, t}^{k}}{2 R_{d k}}\right)^{2} \leq-\frac{P_{d l, t}^{k}}{n_{k} R_{d k}}+\left(\frac{V_{d R, t}^{k}}{2 R_{d k}}\right)^{2}$ & $4.835745 \times 10^{-11}$ & $2.267920 \times 10^{-5}$ & $9.408987 \times 10^{-6}$ \\
$\left(P_{d, t}^{k}\right)^{2}+\left(Q_{d, t}^{k}\right)^{2} \leq\left(S_{d, t}^{k}\right)^{2}$ & $2.11384 \times 10^{-15}$ & $1.164771 \times 10^{-6}$ & $7.966617 \times 10^{-6}$ \\
$Y_{i j, t}^{2}+Z_{i j, t}^{2} \leq X_{i, t} X_{j, t}$ & $1.939844 \times 10^{-10}$ & $3.14378 \times 10^{-5}$ & $5.293519 \times 10^{-5}$ \\
\hline
\end{tabular}

In summary, the proposed MISOCP algorithm for OPD of AC-DC parallel transmission channels could effectively reduce the network losses and improve the economic benefits of the AC-DC interconnected power grid. The computational time of solving the MISOCP model was significantly reduced compared with the computational time of solving the original MINNP model. Moreover, the solution of the proposed MISOCP algorithm had high precision compared with the original MINNP algorithm.

\section{Conclusions}

In this paper, a dynamic OPF model for the day-ahead OPD of AC-DC parallel transmission channels is established. This model can be applied to the formulation of the transmission power schedule of each DC or AC transmission channel. Through the SOC relaxation transformation and the big M method, the OPD model of the AC-DC parallel transmission channels was transformed from a MINNP model to a MISOCP model, which could be tractably solved. The OPD of the AC-DC parallel transmission channels scheme obtained by the proposed method can meet the secure constraints of AC transmission lines and sections, and it can effectively reduce the active power loss of the total AC-DC 
parallel transmission channels, which has good economic benefits. The proposed MISOCP model can significantly reduce the computational complexity of the optimization model. Compared with the MINNP method, the MISOCP method can not only obtain a better optimal solution but also reduce the calculation time and improve the calculation reliability for solving the optimization model.

To extend the proposed algorithm to be more practically applied in actual large-scale AC-DC interconnected power grids, the security constraints of the system under N-1 contingencies should be added to the formulation. Additionally, the algorithm needs to be improved to realize the decoupling calculation of each $\mathrm{N}-1$ contingency due to the large scale of the optimization model including the security constraints of multiple $\mathrm{N}-1$ contingencies. In addition, with the rapid development of VSC-HVDC technology, high voltage and large capacity VSC-HVDC lines are increasingly applied in modern power systems. It is necessary to update the OPD model with the operation constraints of VSC-HVDC lines so that the algorithm is suitable for modern AC-DC interconnected power grids.

Author Contributions: Formal analysis, Z.Y.; Investigation, S.H., Z.T., and Y.S.; Methodology, S.L., Z.Y. and M.L.; Supervision, S.L. and M.L.; Writing-review \& editing, G.F.

Funding: This research was funded by the National Basic Research Program of China (973 Program) (grant no. 2013CB228205), the National Natural Science Foundation of China (grant no. 51207056).

Acknowledgments: The authors gratefully acknowledge the support of the National Natural Science Foundation of China (51977080), and the Fundamental Research Funds for the Central Universities of China (2019MS015).

Conflicts of Interest: The authors declare no conflict of interest.

\section{References}

1. Qin, X.; Zeng, P.; Zhou, Q.; Dai, Q.; Chen, J. Study on the development and reliability of HVDC transmission systems in China. In Proceedings of the 2016 IEEE International Conference on Power System Technology, Wollongong, NSW, Australia, 28 September-1 October 2016; pp. 1-6.

2. Zhou, B.; Rao, H.; Wu, W.; Wang, T.; Hong, C.; Huang, D.; Yao, W.; Su, X.; Mao, T. Principle and Application of Asynchronous Operation of China Southern Power Grid. IEEE J. Emerg. Sel. Top. Power Electron. 2018, 6, 1032-1040. [CrossRef]

3. Shu, Y.; Chen, G.; Yu, Z.; Zhang, Z.; Wang, C.; Zheng, C. Characteristic analysis of UHVAC DC hybrid power grids and construction of power system protection. CSEE J. Power Energy Syst. 2017, 3, 325-333. [CrossRef]

4. Huang, Z.; Guan, L.; Chen, X.; Shen, P.; Zhang, J. Study on a coordinate control strategy of multi-HVDC links to improve transient stability in China Southern Power Grid. In Proceedings of the 2016 IEEE PES Asia-Pacific Power and Energy Engineering Conference, Xian, China, 25-28 October 2016; pp. 1337-1342.

5. Kılıç, U.; Ayan, K. A new approach for optimal reactive power flow solution of multi-terminal HVDC systems using genetic algorithm. In Proceedings of the 2013 3rd International Conference on Electric Power and Energy Conversion Systems, Istanbul, Turkey, 2-4 October 2013. [CrossRef]

6. Nie, Y.; Du, Z.; Li, J. AC-DC optimal reactive power flow model via predictor-corrector primal-dual interior-point method. IET Gener. Transm. Distrib. 2013, 7, 382-390. [CrossRef]

7. Yu, J.; Yan, W.; Li, W.; Chung, C.Y.; Wong, K.P. An unfixed piecewise optimal reactive power flow model and its algorithm for AC-DC systems. IEEE Trans. Power Syst. 2008, 23, 170-176. [CrossRef]

8. Yu, J.; Yan, W.; Li, W.; Wen, L. Quadratic models of AC-DC power flow and optimal reactive power flow with HVDC and UPFC controls. Electr. Power Syst. Res. 2008, 78, 302-310. [CrossRef]

9. Xin, H.; Gan, D.; Huang, Z.; Zhuang, K.; Gao, L. Applications of Stability-Constrained Optimal Power Flow in the East China System. IEEE Trans. Power Syst. 2010, 25, 1423-1433.

10. Cao, J.; Yan, Z.; Xu, X.; He, G.; Huang, S. Optimal power flow calculation in AC/DC hybrid power system based on adaptive simplified human learning optimization algorithm. J. Mod. Power Syst. Clean Energy 2016, 4, 690-701. [CrossRef]

11. Aragüés-Peñalba, M.; Alvarez, A.E.; Arellano, S.G.; Gomis-Bellmunt, O. Optimal power flow tool for mixed high-voltage alternating current and high-voltage direct current systems for grid integration of large wind power plants. IET Renew. Power Gener. 2015, 9, 876-881. [CrossRef]

12. Kılıç, U.; Ayan, K. Optimal power flow solution of two-terminal HVDC systems using genetic algorithm. Electr. Eng. 2014, 96, 65-77. [CrossRef] 
13. Cao, J.; Yan, Z.; He, G. Application of Multi-Objective Human Learning Optimization Method to Solve AC/DC Multi-Objective Optimal Power Flow Problem. Int. J. Emerg. Electr. Power Syst. 2016, 17, 327-337. [CrossRef]

14. Li, Q.; Liu, M.; Liu, H. Piecewise Normalized Normal Constraint Method Applied to Minimization of Voltage Deviation and Active Power Loss in an AC-DC Hybrid Power System. IEEE Trans. Power Syst. 2015, 30, 1243-1251. [CrossRef]

15. Jabr, R.A. Radial Distribution Load Flow Using Conic Programming. IEEE Trans. Power Syst. 2006, 21, 1458-1459. [CrossRef]

16. Farivar, M.; Low, S.H. Branch Flow Model: Relaxations and Convexification-Part II. IEEE Trans. Power Syst. 2013, 28, 2565-2572. [CrossRef]

17. Bai, Y.; Zhong, H.; Xia, Q.; Kang, C.; Xie, L. A decomposition method for network-constrained unit commitment with AC power flow constraints. Energy 2015, 88, 595-603. [CrossRef]

18. Taylor, J.A.; Hover, F.S. Conic AC transmission system planning. IEEE Trans. Power Syst. 2013, 28, 952-959. [CrossRef]

19. Bai, Y.; Zhong, H.; Xia, Q.; Wang, Y. A Conic Programming Approach for Optimal Transmission Switching Considering Reactive Power and Voltage Security. In Proceedings of the 2015 IEEE Power \& Energy Society General Meeting, Denver, CO, USA, 26-30 July 2015; pp. 1-5.

20. Ding, T.; Li, C.; Yang, Y.; Blaabjerg, F.; Zhang, Y.; Yan, H. Second-order cone programming relaxation-based optimal power flow with hybrid VSC-HVDC transmission and active distribution networks. IET Gener. Transm. Distrib. 2017, 11, 3665-3674. [CrossRef]

21. Baradar, M.; Hesamzadeh, M.R.; Ghandhari, M. Second-Order Cone Programming for Optimal Power Flow in VSC-Type AC-DC Grids. IEEE Trans. Power Syst. 2013, 28, 4282-4291. [CrossRef]

22. Bi, R.; Lin, T.; Chen, R.; Ye, J.; Zhou, X.; Xu, X. Alleviation of post-contingency overloads by SOCP based corrective control considering TCSC and MTDC. IET Gener. Transm. Distrib. 2018, 12, 2155-2164. [CrossRef]

23. Kundur, P. Power System Stability and Control; Electric Power Research Institute; McGraw-Hill, Inc.: New York, NY, USA, 1994.

24. Lin, C.; Wei, H.; Hou, W.; Tan, J. Linear inequalities convex transformation for optimal reactive power flow model based on MISOCP relaxations. IET Gener. Transm. Distrib. 2018, 12, 1589-1594. [CrossRef]

25. Gurobi. Available online: https://www.gams.com/latest/docs/S_GUROBI.html (accessed on 1 July 2019). 